\title{
Laminar-to-turbulent transition of pipe flows through puffs and slugs
}

\author{
MINA NISHI I B ÜLENT ÜNSAL ${ }^{1}$, FRANZ DURST ${ }^{1}$ AND \\ GAUTAM BISWA ${ }^{2}$ \\ ${ }^{1}$ Institute of Fluid Mechanics, Friedrich-Alexander Universität Erlangen-Nürnberg, \\ Cauerstrasse 4, D-91058 Erlangen, Germany \\ mnishi@1stm.uni-erlangen.de \\ ${ }^{2}$ Department of Mechanical Engineering, Indian Institute of Technology Kanpur, \\ Kanpur - 208016, India \\ gtm@iitk.ac.in
}

(Received 16 August 2007 and in revised form 7 July 2008)

Laminar-to-turbulent transition of pipe flows occurs, for sufficiently high Reynolds numbers, in the form of slugs. These are initiated by disturbances in the entrance region of a pipe flow, and grow in length in the axial direction as they move downstream. Sequences of slugs merge at some distance from the pipe inlet to finally form the state of fully developed turbulent pipe flow. This formation process is generally known, but the randomness in time of naturally occurring slug formation does not permit detailed study of slug flows. For this reason, a special test facility was developed and built for detailed investigation of deterministically generated slugs in pipe flows. It is also employed to generate the puff flows at lower Reynolds numbers. The results reveal a high degree of reproducibility with which the triggering device is able to produce puffs. With increasing Reynolds number, 'puff splitting' is observed and the split puffs develop into slugs. Thereafter, the laminar-to-turbulent transition occurs in the same way as found for slug flows. The ring-type obstacle height, $h$, required to trigger fully developed laminar flows to form first slugs or puffs is determined to show its dependence on the Reynolds number, $R e=D U / v$ (where $D$ is the pipe diameter, $U$ is the mean velocity in the axial direction and $v$ is the kinematic viscosity of the fluid). When correctly normalized, $h^{+}$turns out to be independent of $R e_{\tau}$ (where $h^{+}=h U_{\tau} / \nu, R e_{\tau}=D U_{\tau} / \nu$ and $U_{\tau}=\sqrt{\tau_{w} / \rho} ; \tau_{w}$ is the wall shear stress and $\rho$ is the density of the fluid).

\section{Introduction}

Since the famous work of Reynolds (1883), it has been well known that all phenomena connected to the laminar-to-turbulent transition of pipe flows are intermittent. If the Reynolds number is high enough so that slugs form, a finite development length in the pipe is required for the flow to go from its laminar into its turbulent state. Over this length, the flow loses its strongly intermittent nature and turns into a fully developed turbulent pipe flow. For a constant pipe length, one observes that the flow intermittency goes from 0 to 1 over a small but finite Reynolds number range, where the intermittency is defined by the ratio of the time summation of the disturbed flow and the total measuring time. 
After the initial work by Reynolds (1883), there were numerous other experimental studies, carried out e.g. by Rotta (1956), Lindgren (1969), Wygnanski \& Champagne (1973), Wygnanski, Sokolov \& Friedman (1975), Rubin, Wygnanski \& Haritonidis (1980), Darbyshire \& Mullin (1995), Draad, Kuiken \& Nieuwstadt (1998) and Hof, Juel \& Mullin (2003), showing that two distinct turbulent flow structures determine the intermittent laminar-to-turbulent transition process. Whether one or the other structure occurs is dependent on the Reynolds number and on the flow disturbance introduced. The flow structures are known as puffs (a low Reynolds number phenomenon) and slugs (a high Reynolds number phenomenon).

The above-mentioned studies support the contention that the laminar-toturbulent transition of pipe flows takes place in the form of puff structures for $R e=D U / \nu \leqslant 2500$, and slug structures for $R e \gtrsim 3000$, e.g. as reported by Wygnanski \& Champagne (1973). The results of their experimental studies showed that puff structures travel with a velocity close to the cross-sectional-area-averaged flow and their centreline velocity at the front edge does not have a clear boundary. As the experiments show, the velocity at the front edge of a puff flow structure changes gradually but at its back edge the velocity changes quite suddenly, showing a welldefined change from the laminar to the turbulent region of the flow. Wygnanski et al. (1975) initiated the puffs using a 1-mm diameter jet produced by a driven loudspeaker and emanating from the wall at the pipe entrance. Rubin et al. (1980) showed that the puff is independent of the type of device that produces the disturbance and its streamwise location, since the basic requirement to initiate the production of turbulence is the creation of a laminar-to-turbulent interface with a large velocity drop across it. The shortcoming of jet triggering of puffs, the large variation in the mass flow rate during the operation of the large disturbance, was removed by the employment of orifice plates at the pipe entry by Bandyopadhyay (1986).

As mentioned earlier, puffs occur at relatively low Reynolds numbers $(R e \lesssim 2500)$ and they are isolated patches of turbulent flow, which are advected downstream with a velocity close to the bulk velocity. The puff flow structure extends 5-20 pipe diameters, depending on the Reynolds number. The transition regions between the laminar flow (outside the puff) and the turbulent flow (within the puff), at the two edges of the puff, are called the front edge and the back edge.

The above investigations clearly show that there is a different type of turbulent structure called a slug. The slug structures have different velocities at their front and back edges. Their front edge travels faster than the cross-sectional-area-averaged flow velocity and also faster than their back edge, so that the lengths of slugs increase as they move downstream in a pipe. Furthermore, slug structures have, within themselves, turbulence properties similar to those of fully developed turbulent flow.

Darbyshire \& Mullin (1995), Hof et al. (2003), Mullin \& Peixinho (2006) and Peixinho \& Mullin (2007) introduced jet disturbances into fully developed pipe flow. They concluded that a critical amplitude of disturbance was needed to cause a transition and it depends on the Reynolds number. Durst \& Ünsal (2006) highlighted the influence of the disturbed wall fence height and the results showed a dependence of critical Reynolds number on the normalized height of their disturbances.

On the basis of the recent discovery of unstable travelling waves in computational studies of the Navier-Stokes equations, Kerswell (2005) explains, in his review, that the travelling wave solutions are the saddle points in phase space. These represent the lowest level in a hierarchy of spatio-temporal periodic flow solutions which can possibly be used to explain the transition to turbulent pipe flows. Faisst \& Eckhardt (2004) by numerical calculation and Hof et al. (2003) by experiments found that the 
turbulent lifetimes start to increase rapidly for $R e \geqslant 1800$ and follows an exponential law with increasing Reynolds number. Peixinho \& Mullin (2006) studied the decay of the puffs with an alternative approach that used sudden decrease of the Reynolds number after disturbing the flow. They discovered that $R e \approx 1750 \pm 10$ is a kind of threshold for lower Reynolds number behaviour and Willis \& Kerswell (2007) estimated this Reynolds number by direct numerical simulation for $R e \approx 1860$. Eckhardt et al. (2007) determined that between the laminar and turbulent states there exists no intermediate state with definite spatial or temporal characteristics. There can be states of the flow dominated by large-scale features that qualify as coherent structures. Nevertheless, the turbulent patches are much larger than the typical wavelengths of the coherent structures.

The present study follows the work reported by Durst \& Ünsal (2006) with a detailed investigation of the development and transport of puffs and slugs from the pipe inlet to the pipe outlet. The present investigations use a test facility that is an extended version of the test facility of Durst \& Ünsal (2006). They performed hot-wire velocity measurements at the end of an $L / D=666.7$ long pipe, where $L$ is the pipe length and $D$ is the pipe diameter, and carried out pressure measurements over the entire pipe length. In their experiments, the laminar-to-turbulent transition occurred naturally at $R e \approx 13000$. Through ring obstacles of different heights, placed at the pipe inlet, the transitional Reynolds number was decreased from 13000 to approximately 2300. The transitional flow structures were formed as puffs for $2300 \lesssim R e \lesssim 3000$ and in the form of slugs for $3000 \lesssim R e \lesssim 13000$. A dimensionless ring obstacle height $h^{+}=h U_{\tau} / v$ (where $U_{\tau}=\sqrt{\tau_{w} / \rho}, \tau_{w}$ is the shear stress at the pipe wall and $\rho$ the density of the fluid) was introduced to characterize the flow disturbance needed for transition as a function of Reynolds number and the measurement results showed a nearly linear dependence of the critical Reynolds number for the range $3000 \lesssim R e \lesssim 13000$ and a strong nonlinear relationship for the range $2300 \lesssim R e \lesssim 3000$. The parameter was computed for many $U_{\tau}$ values for the fully developed pipe flow. Pressure measurements were conducted along the pipe, permitting an insight into the development of the slug flow structures downstream of the pipe inlet.

To trigger single puff and slug structures, Durst \& Ünsal (2006) also employed an electrically driven iris diaphragm, which created instantaneous small flow blockages in the pipe inlet for closing durations of down to $10 \mathrm{~ms}$. This triggering technique permitted the repeatable generation of single puff- and slug-like flow structures in the Reynolds number region where natural transition did not occur. Hence, in this way, the device of Durst \& Ünsal (2006) greatly facilitated accurate measurements of the front and back edge velocities of puffs and slugs through the installed pressure sensors. Phase-resolved velocity measurements with a hot-wire sensor, located at the end of the pipe, were also performed to gain a deeper insight into the flow structures of slugs and puffs. These latter measurements were qualitative in nature in the work of Durst \& Ünsal (2006) and did not aim to give the detailed characteristics of the single structures.

In the present work, the 'iris diaphragm single flow structure triggering technique' was used to investigate the dynamics of the puff- and slug-structure development, from their generation at the pipe inlet over the entire pipe length. The measurements were conducted for different pipe lengths starting from $0.5 \mathrm{~m}$ and extending to $8 \mathrm{~m}$. The pipe diameter was kept constant at $15 \mathrm{~mm}$. For every pipe length, the occurrences of flow structures and their development were investigated by hot-wire velocity measurements. The change in the puff structures, with increasing Reynolds number, to slug structures could be shown through phase-averaged velocity measurements. 


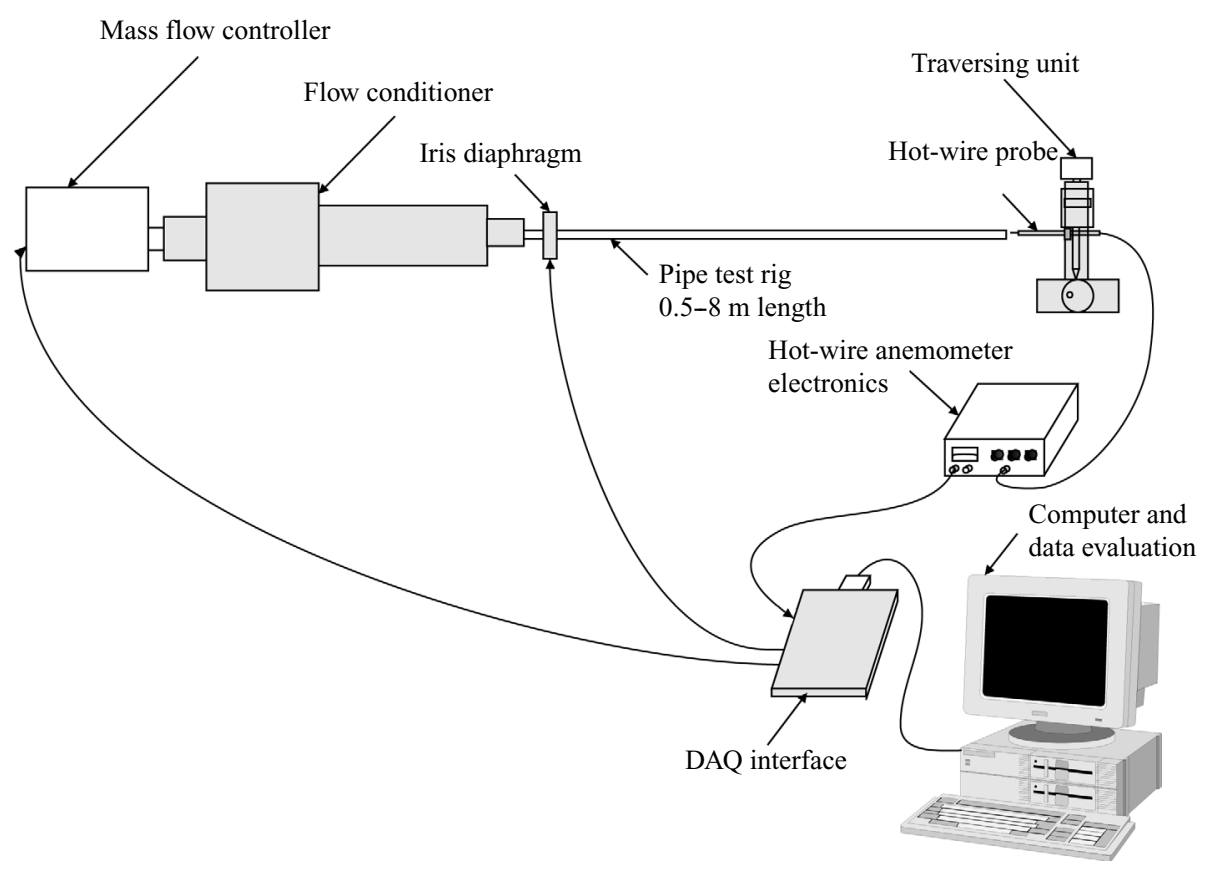

FIGURE 1. Schematic representation of the experimental test rig, triggering device and measuring equipment.

The splitting of puff structures, starting at a certain Reynolds number and at various pipe lengths, is shown from velocity signals obtained at the centre of the pipes. This phenomenon occurred at Reynolds numbers that were not high enough to produce the slugs directly. The investigations showed that the split puffs propagate without changing their form and duration at a certain Reynolds number.

A final set of measurements was also carried out to trigger the laminar-to-turbulent transition of fully developed laminar pipe flows. For this purpose, ring-type obstacles of predetermined height were employed and for each height the critical Reynolds number was determined. There are different possible definitions of the start of transition, e.g. according to the changes in root mean square values (Durst \& Ünsal 2006) while for the present investigation, it is defined by the first occurrence of puffs. The results show that $h^{+}\left(R e_{\tau}\right)=$ constant holds for the triggered laminar-to-turbulent transition of fully developed laminar pipe flows.

The plan of the paper is as follows. In $\S 2$, the test rig, the triggering device and measurement technique are briefly outlined. Section 3 describes the verification of the experiments and validation with the earlier experiments of Durst \& Ünsal (2006). Section 4 details the development of slug structures. Section 5 discusses the development of puffs and the puff structure. Section 6 summarizes the effect of triggering at a location where the flow is fully developed. The conclusions and the outlook for future research are presented in $\S 7$.

\section{Test rig, triggering device and measurement equipment}

The experimental set-up, including the mass-flow-rate control unit (see figure 1), used in the present investigations, was explained in detail by Durst et al. (2003) and Durst \& Ünsal (2006). Therefore, only a brief description will be given here for 


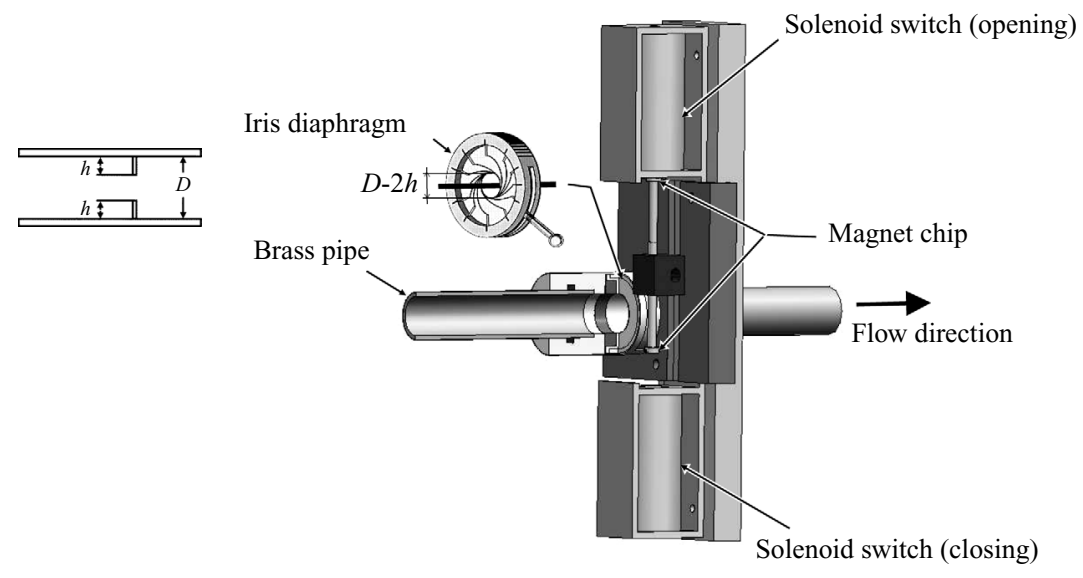

FIGURE 2. Three-dimensioned presentation of the iris diaphragm triggering system.

the sake of completeness and to provide a clear understanding of the experimental equipment employed.

The present parts of the test rig, shown in figure 1, were the mass-flow-rate control unit (MFCU), the pipe flow test section, shown together with a flow conditioner (plenum chamber with honeycomb structures and an inlet nozzle) at the inlet of the pipe, the hot-wire anemometer system and the computer operated with a special data acquisition system. This allowed different Reynolds numbers to be set and the preset flow rate to be maintained for each of the investigations. Due to the critical valve operation principle of the MFCU (see Durst et al. 2003), the pressure changes in the pipe, induced by the turbulence structures during an intermittent transition process, did not affect the set mass-flow-rate value. Hence, the employment of the MFCU ensured constant Reynolds number operation of the test rig, in spite of the occurrence of the laminar-to-turbulent transition of the investigated pipe flows.

To trigger single puff and slug structures in pipe flows, an electrically driven iris diaphragm unit, shown in figure 2, was employed. Two magnets were used to achieve independently controlled closing and opening of the iris diaphragm. The height of the iris diaphragm after closing was adjusted to yield predefined heights at the wall to trigger the flow in accordance with the findings of Durst \& Ünsal (2006) regarding the critical ring obstacle height.

The duration of operation of the iris diaphragm was controlled electrically through a driving signal imposed on the triggering device by the computer employed. The smallest closing and opening duration that could be realized with the added magnets was $30 \mathrm{~ms}$, which was small enough to generate repeatable single puff and slug structures in all experiments. Therefore, the effect of a lapse duration shorter than $30 \mathrm{~ms}$ is not known, although if the lapse time exceeds a certain value, the iris diaphragm obstacle would function as a ring-type obstacle, which means that it disturbs the flow continuously, and as a consequence one obtains two or more disturbed structures combined, which cannot be distinguished as a single structure.

The present experimental investigations were carried out for a pipe of diameter $D=15 \mathrm{~mm}$ and for nine different pipe lengths $(L=0.5,1,2,3,4,5,6,7$ and $8 \mathrm{~m})$. Hot-wire velocity measurements were conducted at each pipe outlet, i.e. for each pipe length, over the entire Reynolds number range $R e \approx 2300-11500$. $(\operatorname{Re} \approx 11500$ 
was the critical Reynolds number $(R e)_{\text {crit }}$ at which the laminar-to-turbulent transition occurred naturally with the present pipe flow.) For each pipe length, the laminar velocity profiles were measured to ensure flow symmetry. The velocity measurements for studying the occurrence of slug- and puff-like flow structures were conducted at the centreline of the pipe. Puff and slug structures, generated through periodical closing and opening of the iris diaphragm, were investigated as functions of time by hot-wire velocity measurements. Approximately 100 puffs and slugs were investigated in each set of experiments so that statistically averaged values, such as the mean velocity and different turbulence quantities, could be measured for each of the Reynolds numbers. The test rig was also employed for transitional flow investigations with fixed height ring-type obstacles mounted as described by Durst \& Ünsal (2006). These experiments are described separately in $\S 6$.

The hot-wire probe employed was mounted on a traversing unit. A single-wire probe, connected to DISA 55 M01 constant-temperature hot-wire anemometer electronics, was used in all the experiments. The traversing system was moved vertically using the computer to change the wire position to measure the velocity for $30 \mathrm{~s}$ at each position to obtain the cross-sectional velocity profile. The traversing system was also used to obtain the time profile of the puffs at different locations. The hot-wire anemometer output was connected to a 16-channel, 16 -bit, $333 \mathrm{kHz}$ data acquisition card for simultaneous measurements of velocities. The input flow rate of the mass-flow-rate controller was also set by the computer and a special software program ensured that all the measurements were carried out in a well-controlled manner. Various sub-programs within the data acquisition software were written to carry out the processing of all data to yield the flow information provided in the present paper.

\section{Verification experiments}

Prior to carrying out studies of puff and slug flow development as a function of pipe length, verification experiments were performed to ensure the correct functioning of the entire test rig, especially the triggering device and the measuring equipment employed. These measurements clearly showed that the test rig permitted the pipe flow to remain laminar up to $R e \approx 11500$ and over the length of $L=8 \mathrm{~m}$ corresponding to $L / D=533.3$ with $D=15 \mathrm{~mm}$. For different pipe lengths, the laminar flow development was checked, yielding detailed cross-sectional velocity profile information. The development of the velocity profiles with pipe length agreed with corresponding flow predictions and confirmed the development length correlation proposed by Durst et al. (2005):

$$
\frac{L}{D}=\left[(0.619)^{1.6}+(0.0567 R e)^{1.6}\right]^{1 / 1.6} .
$$

Hence, for every investigated flow Reynolds number, the development length required to yield a parabolic velocity profile could be computed. This information permitted the state of the flow to be assessed for the investigated pipe lengths, that is the state in which the flow would have been without the introduced intermittent flow disturbances.

The triggering system of the iris diaphragm permitted controlled flow disturbances to be introduced that resulted in puff- or slug-like flow structures for $2300 \lesssim R e \lesssim$ 11500 . To test the triggering system, ring-type obstacles were also employed to obtain information on the dependence of the transition Reynolds number of the 


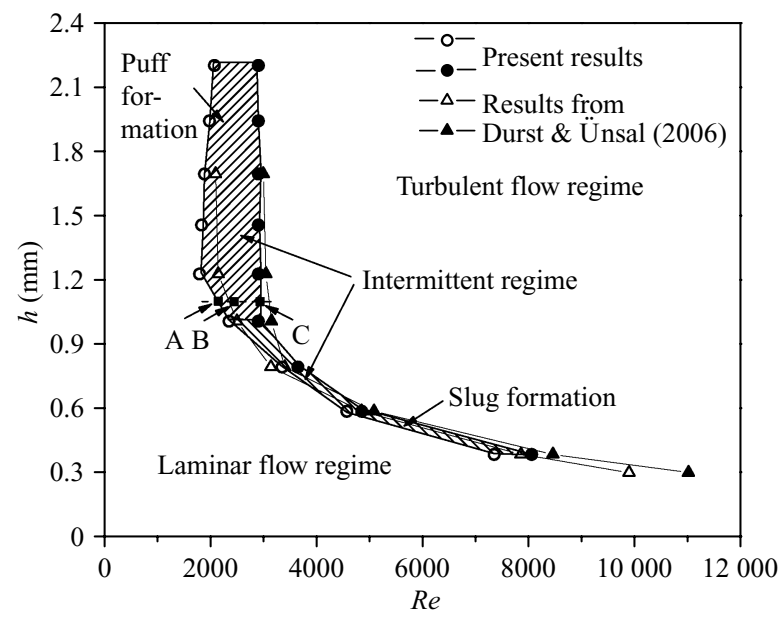

FIGURE 3. Dependence of critical Reynolds number on the normalized ring fence height.

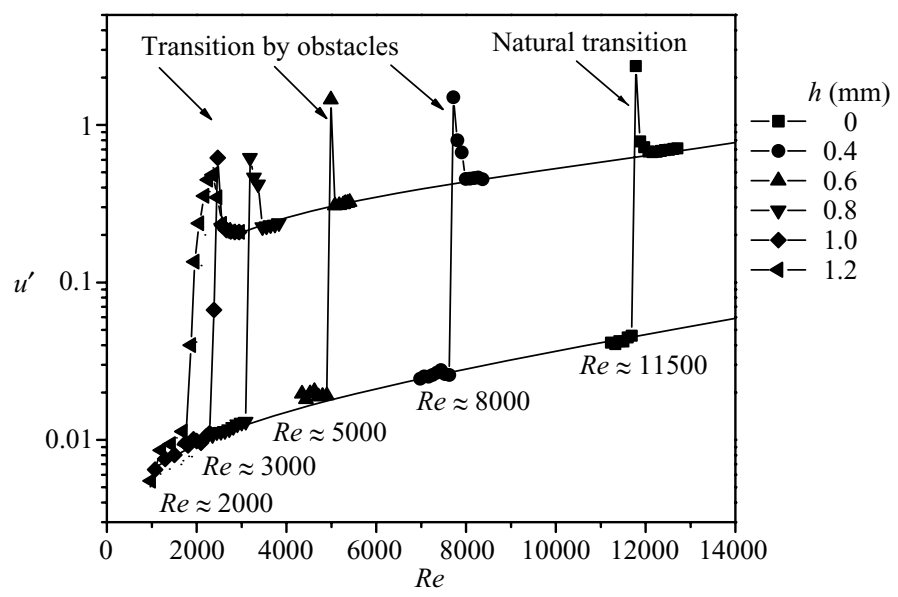

FIGURE 4. Natural transition and transition by ring fence obstacles.

flow on their height $h$ for $1800 \lesssim R e \lesssim 11500$. For low Reynolds numbers with large triggering ring obstacle heights $h$ (see figure 3), puff-like flow structures resulted. For higher Reynolds numbers, only slug-like flow structures were found. This is shown in figure 3, which provides information on the triggering height $h$ required as a function of Reynolds number to yield the laminar-to-turbulent transition of pipe flows.

The results in figure 3 agree fairly well with those presented by Durst \& Ünsal (2006), who also performed measurements with carefully manufactured ring fence obstacles. The small differences recorded for the region of puff formation, caused by the difference in definition of the critical Reynolds number, are not of any importance for the studies described in this paper.

Figure 4 shows the dependence of the critical Reynolds number on the wall height $h$ by plotting the time-averaged $u^{\prime}$ value versus Reynolds number. The $u^{\prime}$ value is defined as $u^{\prime}=\sum_{t=0}^{T} \sqrt{\left(\bar{U}-u_{t}\right)^{2}} / N$, where $\bar{U}$ is the average velocity in time $T, u_{t}$ is the instantaneous velocity and $N$ is the number of measurement points in time $T$. The sudden jump of the $u^{\prime}$ value at a certain Reynolds number indicates that the transition 


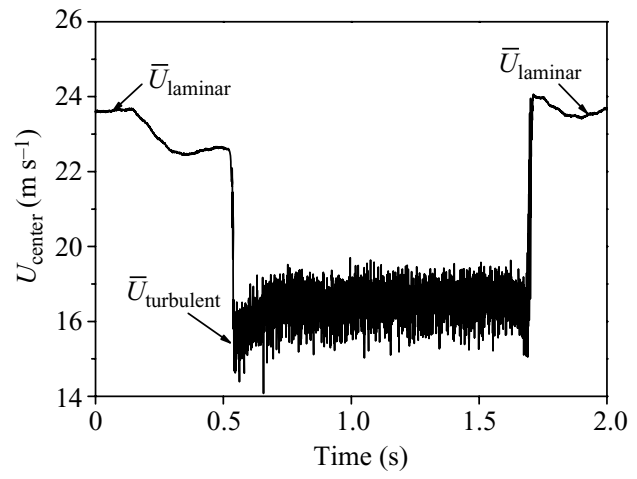

FIGURE 5. Single slug signal of natural transition at $R e=11500$.
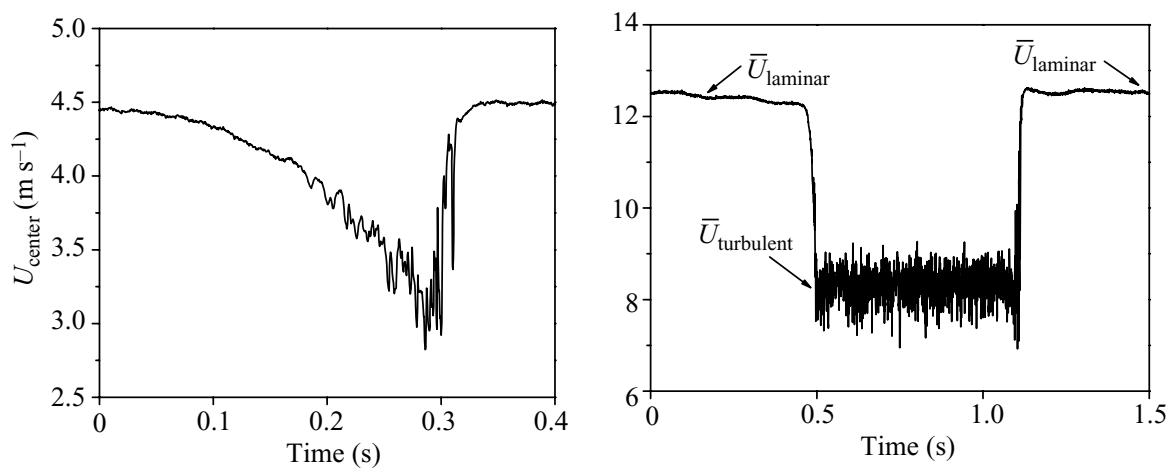

FIgURE 6. Puff (left) and slug (right) signals initiated by the iris diaphragm triggering device.

occurred. The iris diaphragm triggering device is able to provide arbitrary occurrence of puffs and slugs: the open-close timing and opening area of the iris diaphragm were changed from a minimum to a maximum value. In this way, the transition usually observed for the natural laminar-to-turbulent transition (see figure 4) could be studied at every chosen Reynolds number $\left[R e<(R e)_{\text {crit }}\right]$ by adjusting the iris diaphragm height accordingly. This suggests that the test rig with the iris diaphragm triggering device is very well suited to study laminar-to-turbulent transition in pipe flows. The control provided is well suited for detailed experimental flow investigations of otherwise uncontrolled flow phenomena.

For Reynolds numbers of approximately $R e \approx 11500$, naturally occurring slug flows were recorded for the present test rig and examples are shown in figure 5, which illustrates the typical features already pointed out by Wygnanski \& Champagne (1973). Typical flow structures for $R e \lesssim 2300$ puff flows and for $R e \gtrsim 3000$ slug flows, which were initiated by the iris diaphragm triggering device, are indicated in figure 6 . A comparison of literature data with the traces in figure 6 shows that there is no difference in the forms of puffs and slugs compared with the signals reported by Wygnanski \& Champagne (1973). Furthermore, the velocity records along the pipe permitted the front and back edge propagation velocities of the triggered slugs to be compared with corresponding results available in the literature. Figure 7 shows good agreement between triggered and naturally occurring transitional flow propagation velocities. The comparison was made with the data recorded by both Lindgren (1969) and Wygnanski \& Champagne (1973), who measured the propagation velocities 


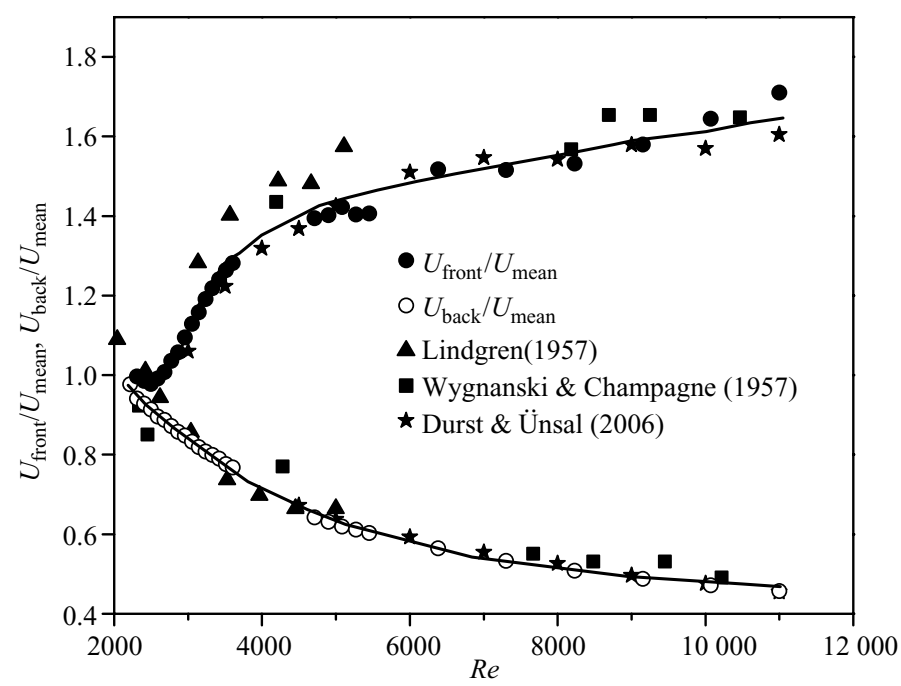

FiguRE 7. Comparison of the present non-dimensional front and back edge propagation velocity of puffs and slugs with previous results in which solid lines were drawn to guide the eye.
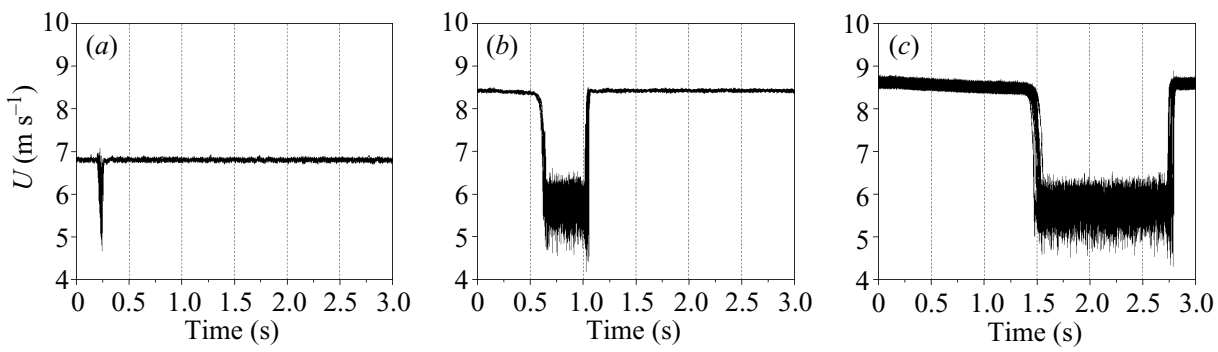

FIGURE 8 . Slug development along the pipe and repeatability at $R e=4160$ :

(a) $0.5 \mathrm{~m},(b) 3 \mathrm{~m},(c) 8 \mathrm{~m}$.

of naturally occurring transitional flow structures, together with the results from Durst \& Ünsal (2006). The data of different studies collapse onto the solid lines shown in figure 7. In fact, the comparisons in the figure confirmed that the present puff and slug structures were similar to those which occur at Reynolds numbers where the laminar-to-turbulent transition occurs naturally, as briefly mentioned by Durst \& Ünsal (2006).

\section{Studies of slug developments}

The test rig, described in $\S 2$ and applied in $\S 3$ for the first experimental studies of laminar-to-turbulent transition of pipe flows, was then extensively applied to study slug flows. Examples of hot-wire velocity measurements at pipe lengths of $L=0.5,3$ and $8 \mathrm{~m}$ are shown in figures $8-10$ for $R e=4160,6250$ and 8230 . Each of the time records shown is composed of 11 individual slugs. The individual slugs in figures $8-10$ cannot be identified any longer, which shows the high degree of reproducibility with which the iris diaphragm triggering device is able to produce slugs.

The shortest time $(30 \mathrm{~ms})$ achievable to close partially and reopen the iris diaphragm was employed for each Reynolds number. Each closing produced a 

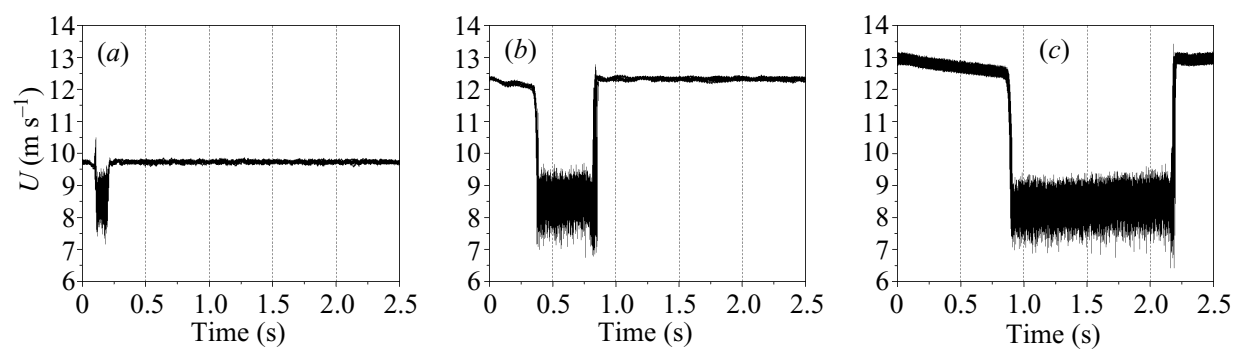

FIGURE 9. Slug development along the pipe and repeatability at $R e=6250$ :

(a) $0.5 \mathrm{~m},($ b) $3 \mathrm{~m},(c) 8 \mathrm{~m}$.
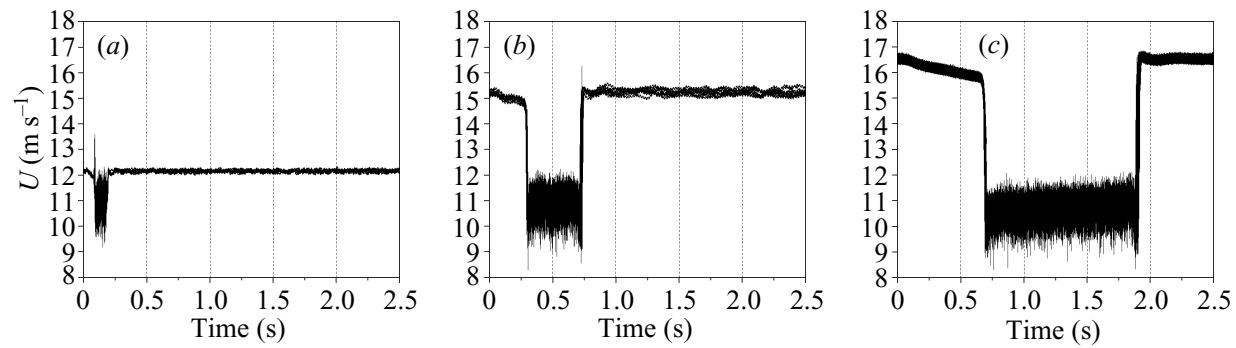

FigURE 10. Slug development along the pipe and repeatability at $R e=8230$ :

(a) $0.5 \mathrm{~m},(b) 3 \mathrm{~m},(c) 8 \mathrm{~m}$.

ring fence disturbance with a height of $h \mathrm{~mm}$, in accordance with results plotted in figure 3 to ensure that the initial disturbances had sufficient strength to form a slug and allow its study as it moves downstream in the pipe. During this motion, the length of the slug is increasing. This is shown in figures 11 and 12 for nine downstream measuring locations and four Reynolds numbers. These figures clearly indicate the different speeds at which the front and the back edges of the slug move. A higher front edge velocity, already indicated in figure 7, can be deduced from these figures.

Figure 13 provides information on the duration of the deterministically produced slugs. The figure shows that the duration $\Delta t_{s}$ of a slug increased with increasing pipe length and that the higher the Reynolds number, the smaller $\Delta t_{s}$, which indicates that the decrease in the duration of the slug was caused by the increase in the velocity. Figure 14 shows the non-dimensionalized propagation velocity of the front and back edge propagation velocity. This is calculated as the time between the iris diaphragm being operated and the slug being detected at the exit of the pipe. The figure shows that both the front and back edge propagation velocities increase drastically with the pipe length between $L=0.5$ and $2 \mathrm{~m}$. This indicates the strong nonlinearity in the propagation velocity of slugs, caused by the effects of both the development to the parabolic velocity profile and the turbulence propagation, especially near the inlet region, $L \leqslant 2 \mathrm{~m}$.

The test rig also permitted sequences of slugs to be produced with time intervals between them, as indicated in figure 15, which shows that changing the iris diaphragm permitted the time intervals between the slug-generating disturbances to be changed. Figures $15(a)$ and $15(b)$ show that a length of the pipe of $L=8 \mathrm{~m}$ was sufficient for the individual slugs with two different intervals to merge. Hence there was one slug-like flow left at the pipe outlet at $L=8 \mathrm{~m}$. In figure $15(\mathrm{c})$, the interval was 

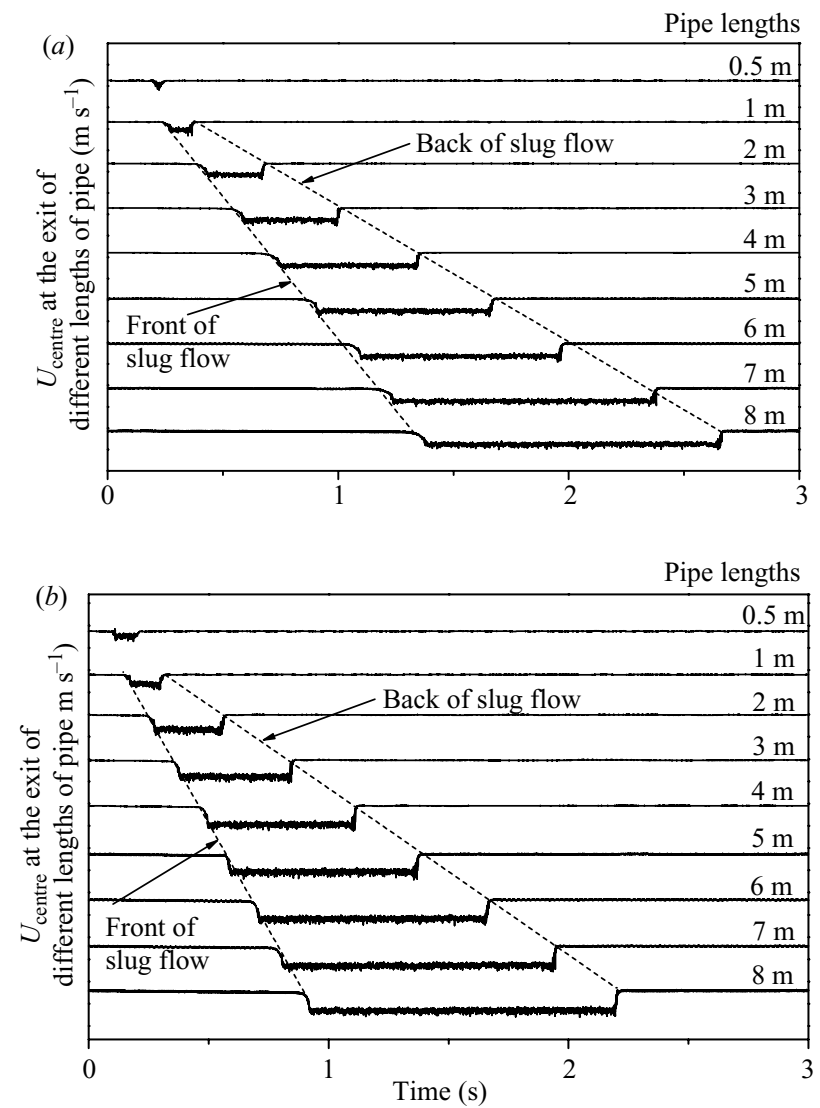

FIGURE 11. Slug development with increasing Reynolds number of different pipe lengths for (a) $R e=4530$ and $(b) R e=6380$.

chosen such that the length of the pipe $L=8 \mathrm{~m}$ was insufficient for the slugs to merge. These slugs would have merged, however, if the pipe length had been larger. Taking a certain length of pipe but at two different locations, the intermittency is higher downstream than upstream. This clearly shows that laminar-to-turbulent transition records show a strong $L / D$ dependence as Rotta (1956) also mentioned in his experimental investigation.

\section{Studies of puff development}

It is apparent that the test facility described in $\S 2$ is also suited to the introduction of disturbances that result in the formation of puffs. For this purpose, the iris diaphragm was closed sufficiently to yield disturbances of about $h \approx 1.1 \mathrm{~mm}$ and the Reynolds number of the flow was changed for this set of experiments from $R e \approx 2300$ to 4300. In this Reynolds number range, the changes in the structures of puffs were studied.

The results of the measurements for Reynolds numbers from $R e \approx 2300$ (point $\mathrm{A}$ in figure 3 ) to $R e \approx 2900$ (point $\mathrm{C}$ in figure 3 ) with different pipe lengths are shown in figures 16 and 17. These show that for the lowest Reynolds number, $R e=2310$ (figure 16a), a single puff formed in the entrance region of the pipe. As it moved downstream with the flow, it was maintained as a single puff. As the Reynolds number 

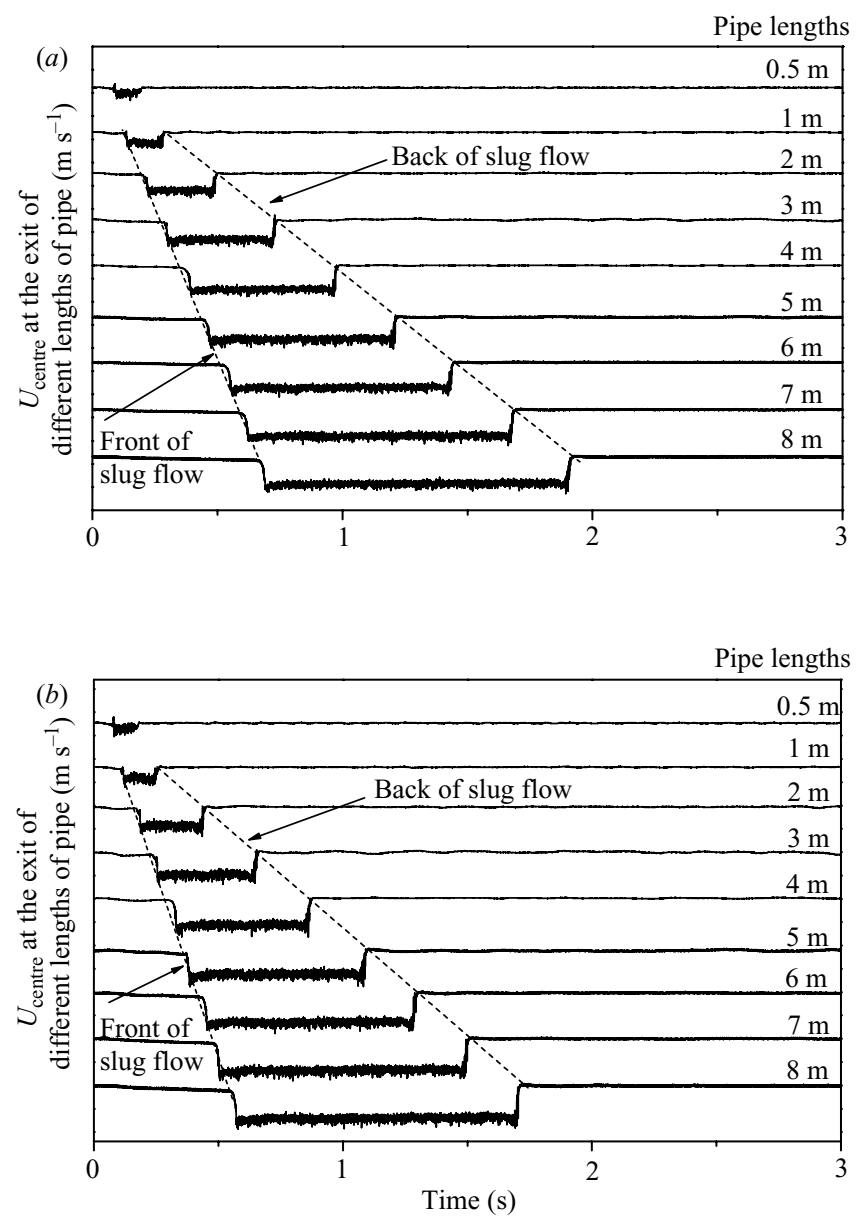

FIGURE 12. Slug development with increasing Reynolds number of different pipe lengths for (a) $R e=8230$ and (b) $R e=10070$.

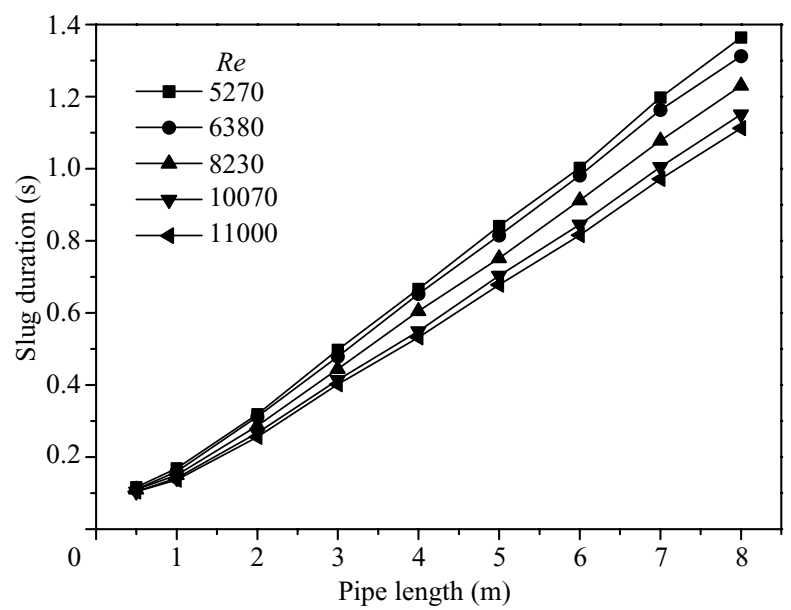

FIGURE 13. Duration of slugs with increasing pipe length. 


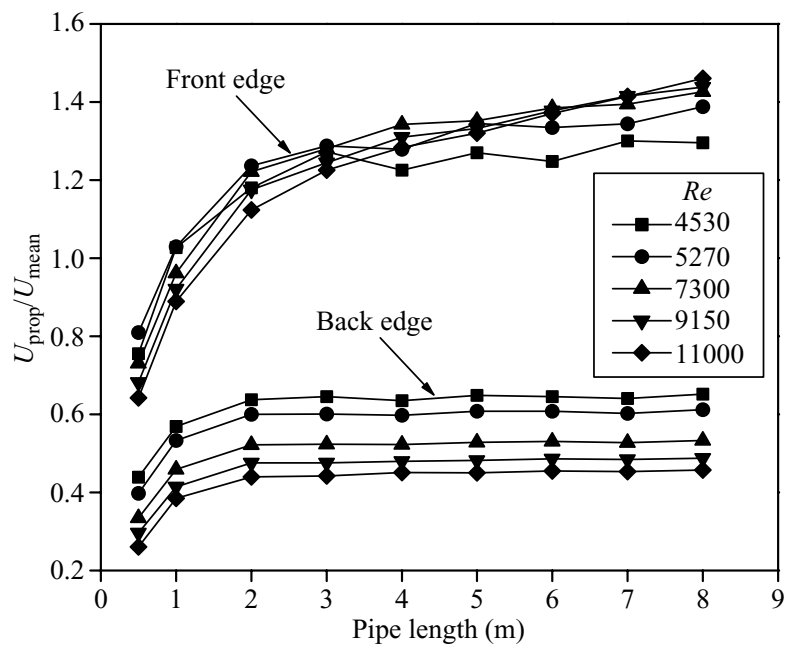

FIGURE 14. Non-dimensionalized slug propagation velocity with increasing pipe lengths for $R e=4530-11000$.

(a)

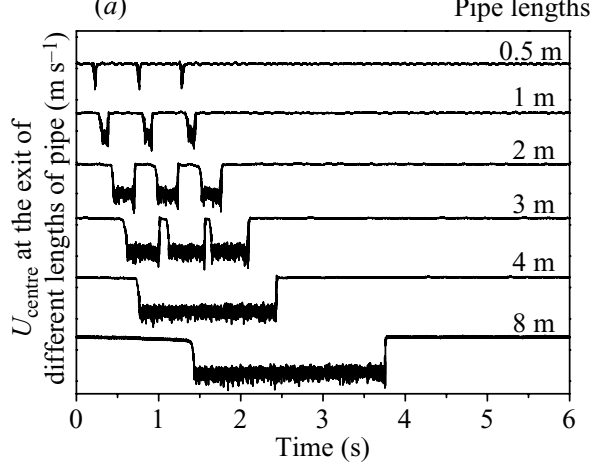

(b)

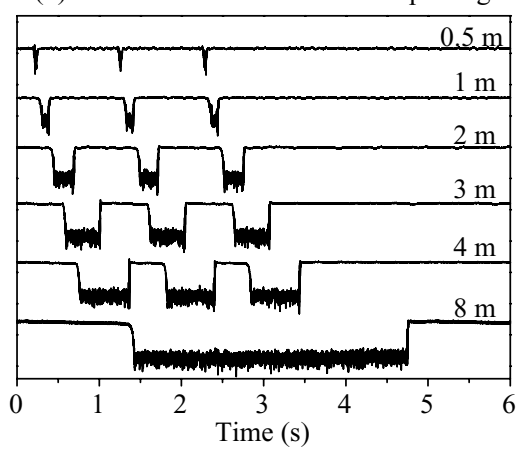

Pipe lengths

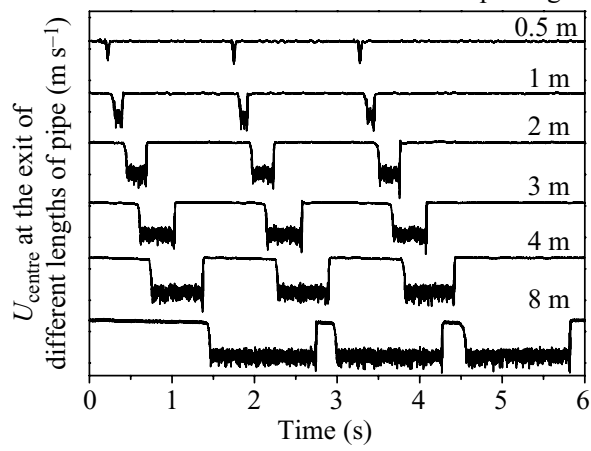

FIGURE 15. Triple shot and slug joining at $R e=4200:(a)$ Interval $=0.5 \mathrm{~s},(b)$ Interval $=1 \mathrm{~s}$, (c) Interval $=1.5 \mathrm{~s}$.

was increased to $R e=2495$ (figure $16 b$ ), corresponding to point B in figure 3, a single puff remained only up to about a pipe length of $L=5 \mathrm{~m}$. Thereafter puff splitting occurred, yielding basically a sequence of two puffs. For even higher Reynolds numbers (see figure 17a), corresponding to point $\mathrm{C}$ in figure 3 , puff splitting was 

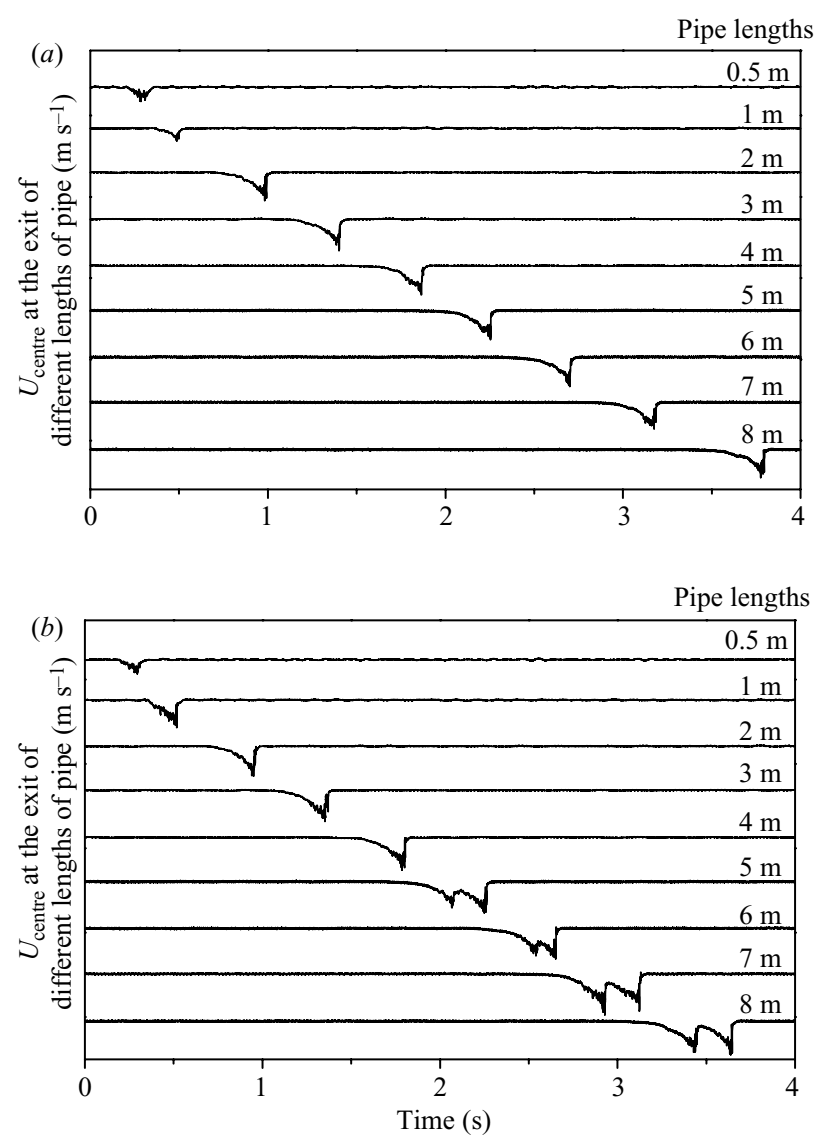

Figure 16. Puff development with increasing Reynolds number of different pipe lengths for (a) $R e=2310$ and $(b) R e=2495$.

observed very much earlier, permitting the flow between the front and the back of the disturbed flow to develop and yielding, further downstream, slug-like flows. The front edge of the resulting flow still had the properties known for puffs, although the structures appear much more like slugs than puffs in figure $17(b)$. The back edge of the resultant flow structure showed already the steep change from the turbulent to laminar state of the flow, characteristic of slugs.

For the smallest Reynolds number investigated in this set of experiments, $R e=2310$, puff average velocity information was obtained as illustrated in figure 18(a), showing the velocity on the pipe axis at $L=8 \mathrm{~m}$ as a function of time. Single puffs with a high repeatability of their velocity fields resulted for this low Reynolds number, with a slow decrease in mean velocity at their front edge and a sudden velocity increase at the back edge. The velocity profiles, provided in figure $18(a)$ as a function of time, are typical for puff-like flow structures in the laminar-to-turbulent transition region of pipe flows at $L=8 \mathrm{~m}$. If one keeps $h \approx 1.1 \mathrm{~mm}$ and increases the Reynolds number of the pipe flow in small steps, the puff-like flow structure changes, as illustrated in figure $18(b-d)$. In figure $18(b)$, typical puff splitting occurs, yielding two velocity peaks. Increasing the Reynolds number further yields velocity distributions over time that are similar to those of slugs (figures $18 c$ and $18 d$ ), at least in the centre part of the structure. Only the front and back edges of the signal seem to differ from those of 

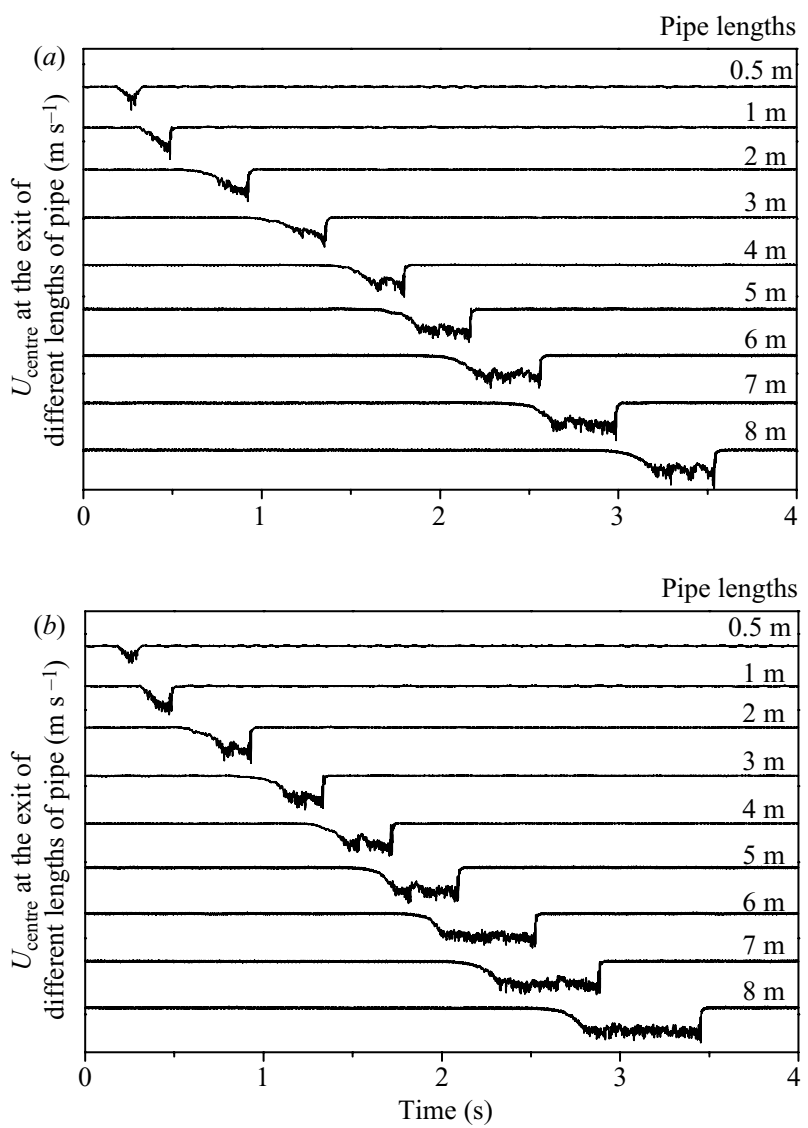

FIGURE 17. Puff development with increasing Reynolds number of different pipe lengths for (a) $R e=2680$ and $(b) R e=2865$.

slug flows. Slugs can also be formed by low height obstacles if the Reynolds number is sufficiently high, that is higher than the Reynolds number indicated by point $\mathrm{C}$ in figure 3.

Figure $19(a-d)$ presents the turbulent intensities corresponding to the cases illustrated in figure $18(a-d)$. Figure $19(a)$ reveals that the turbulent intensity varies from $2 \%$ to $15 \%$ in the case of an equilibrium puff $(R e=2310)$. The split puffs show a similar trend of variation in the intensity (figure 19b). Figures 19(c) and 19(d) show that the structures produced have similar aspects to slug structures; the average turbulent intensities in the middle of the structures are $5 \%$ and $4.5 \%$, respectively, keeping the maximum instantaneous intensity value close to $15 \%$.

The results in figure 20 are examples chosen from a large number of time records for the instantaneous velocities of the puffs at different radial locations $r / R$, where $r$ is the distance from the centre point of the pipe and $R$ is its radius. Each time profile of the axial velocities in the figure is obtained by phase averaging of 60 samplings. The puff structures presented in figures 20 and 21 correspond to $R e=2450$. At the location $r / R=0.93$, the axial velocity does not show a large change even when the velocity at the centreline $(r / R=0)$ reaches the minimum value in figure 20. This complex structure of puffs is discussed by Van Doorne et al. (2007), who visualized puffs by the stereoscopic particle image velocimetry (SPIV) system. They claim that this is 

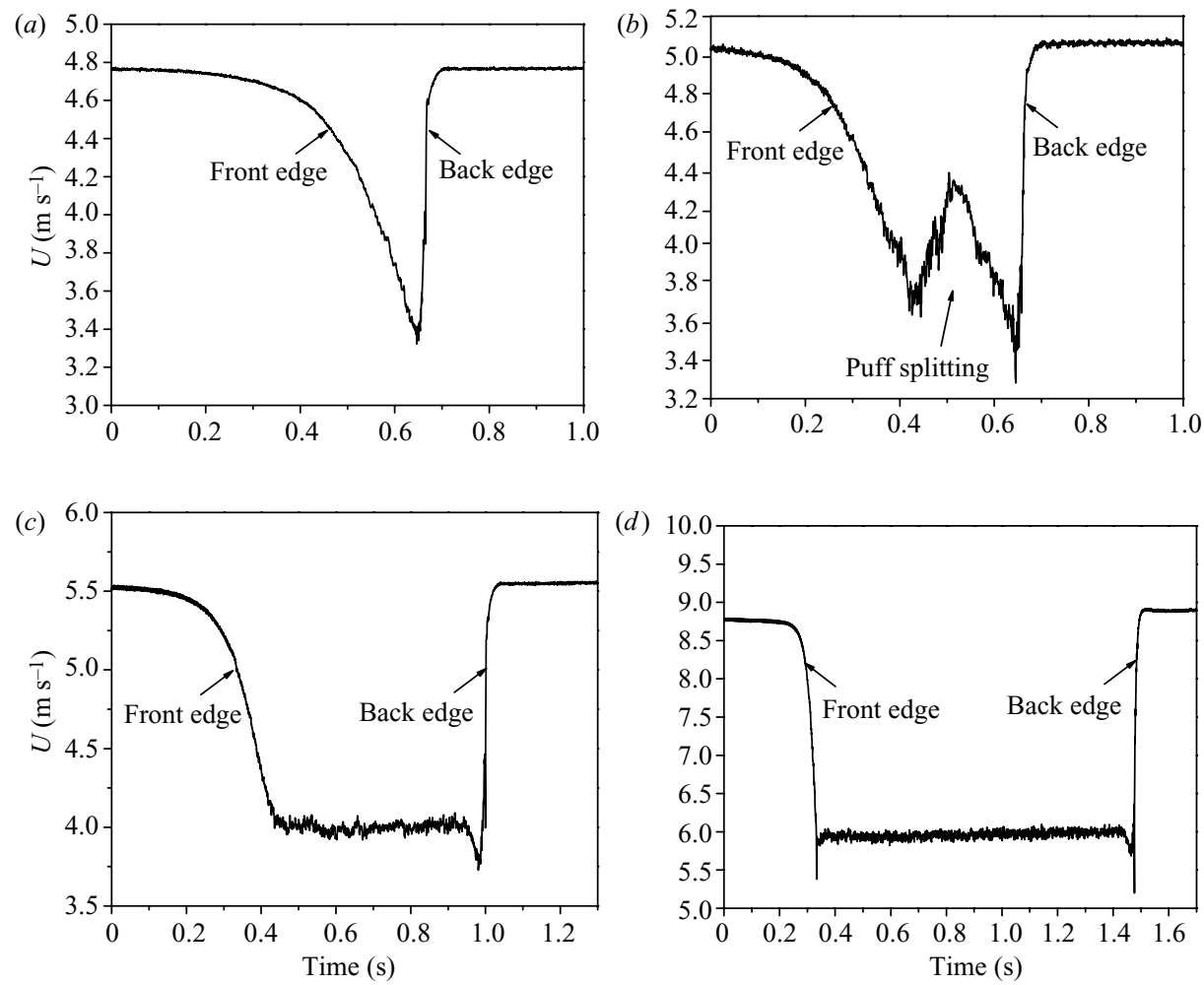

FIGURE 18. Cycle-averaged-axial velocity distribution at the centreline of a puff at (a) $R e=2310$, (b) $R e=2495$, (c) $R e=3235$ and (d) $R e=4345$ at $L=8 \mathrm{~m}$.

an indication of the quasi-periodic regeneration of hairpin vortices. The result shows good agreement with their observations; whereas they took only one puff structure, the present results are ensemble averaged signals. The typical cross-sectional velocity profiles measured at the exit of the pipe for the puff structures at $R e=2450$ are shown in figure 21. The figure shows that the structures do not have a block profile inside whereas slugs and intermediate puffs do, as shown by Durst \& Ünsal (2006). The measurements were carried out for one radius and are shown together in a symmetrical way in the figure for clearer presentation. The structures reveal laminarto-turbulent transition between $t=4$ and $4.40 \mathrm{~s}$. The figure clearly shows the existence of a vortex-like structure near the wall region.

It is interesting that the experimental studies of Wygnanski \& Champagne (1973) resulted in two clearly separated regions for the appearance of slugs and puffs in transitional pipe flow. They only observed puffs for very high levels of disturbances and at low Reynolds numbers. This clear separation between slugs and puffs was not found by Darbyshire \& Mullin (1995), who detected both in some flow regimes. They also found that their mixed occurrence was not just dependent on the magnitude of the disturbance but also on the type of flow disturbances that they introduced. In the present study, the flow disturbance was introduced at the pipe inlet by a short duration of inserted 'wall fences'. For this kind of disturbance, the results in figure 3 were obtained, which indicated clear puff formation only for disturbances located to the left of the marked vertical area, indicating that the introduction of large disturbances was needed. As one moves away from the left-hand side of this 

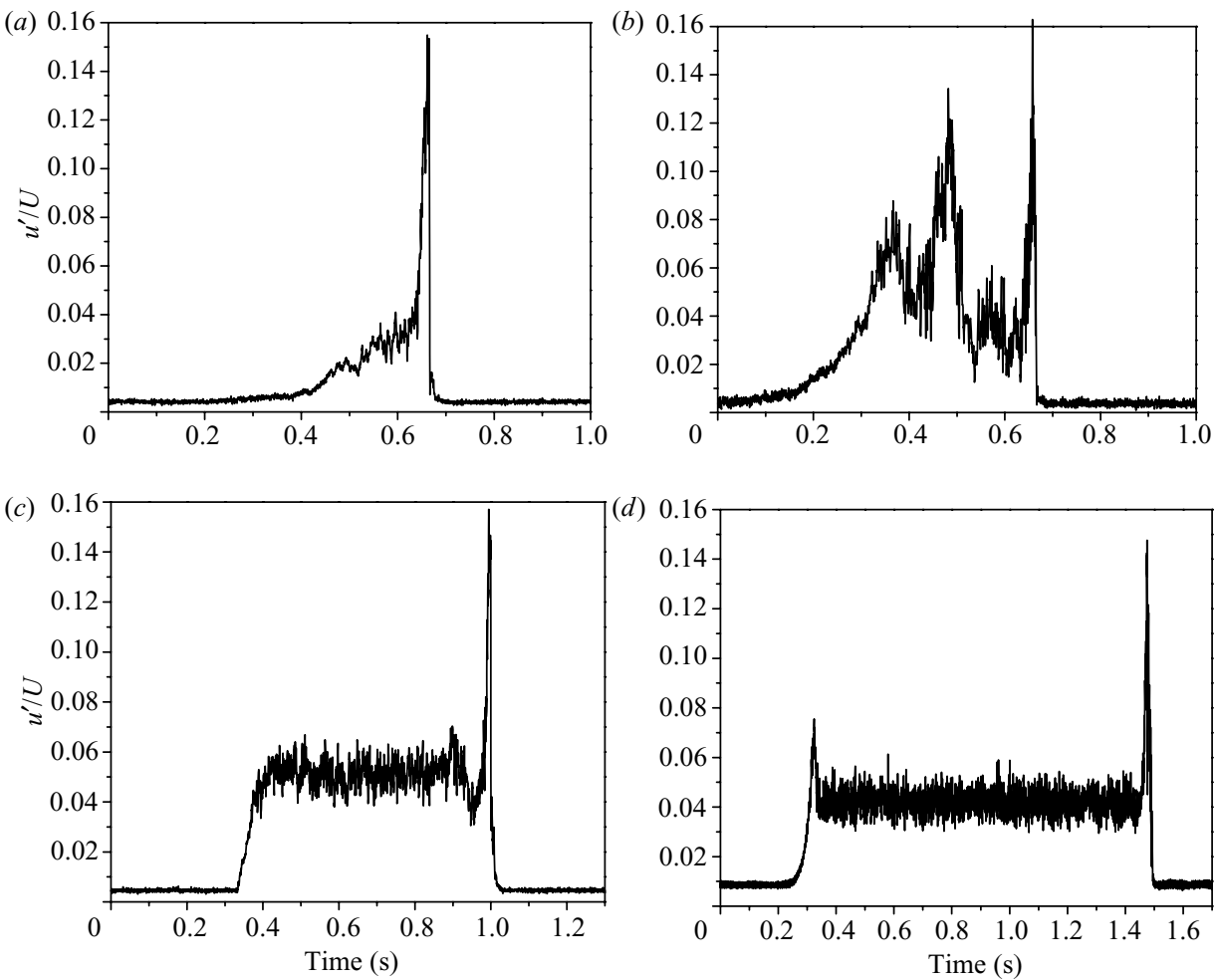

FIGURE 19. Turbulent intensity in the axial velocity signal at the centreline in a puff at (a) $R e=2310$, (b) $R e=2495$, (c) $R e=3235$ and (d) $R e=4345$ at $L=8 \mathrm{~m}$.

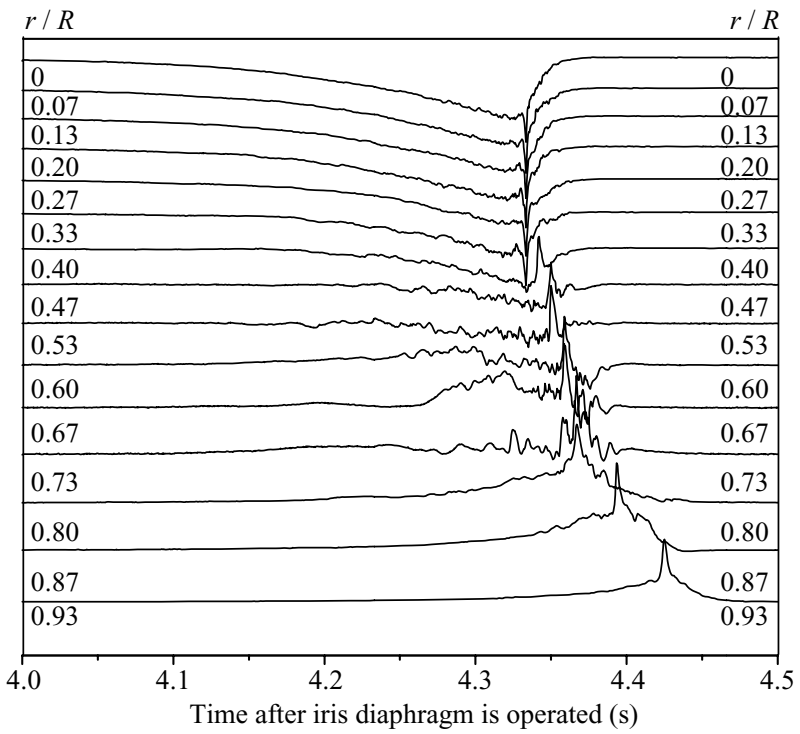

FIgURE 20. Axial velocity at different radial position $r / R$ vs. time which is shown from the time when the iris diaphragm is operated at $R e=2450$. 


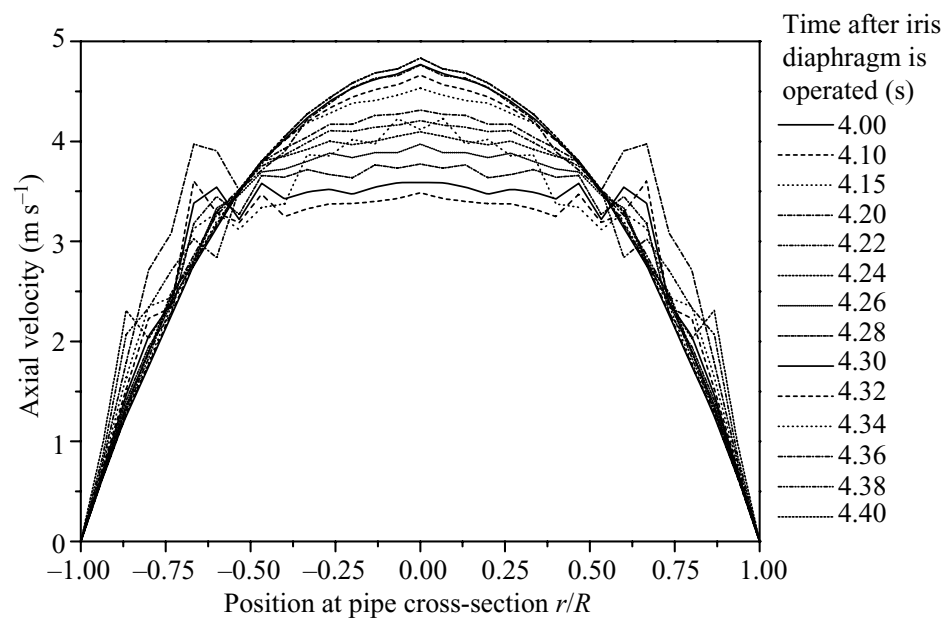

FIGURE 21. Axial velocity as a function of different radial position $r / R$ at different time after the iris diaphragm is operated at $R e=2450$.

area, e.g. with increasing $R e$ from point A, leaving $h$ the same, the puffs that occur split, yielding a sequence of two puffs initially. As the Reynolds number is increased further, multiple splitting seems to occur that yields, finally, flow structures that have an overall appearance similar to that of slugs. Without doubt, the present test rig set-up proved to be well suited for studying phenomena of this kind. Its highly reproducible triggering capabilities make the occurrence of puffs and slugs a deterministic rather than a statistical phenomenon.

\section{Triggering of fully developed pipe flow}

So far, the investigation described has been aimed at a better understanding of the laminar-to-turbulent transition of pipe flows when triggered by an iris diaphragm mounted at the inlet of the pipe. This means that the triggering of individual puffs and slugs by the iris diaphragm device occurred at the same location at the inlet. However, at the inlet of the pipe, in all the investigations described so far, the velocity profiles were dependent on the inlet flow conditions, defined by the plenum chamber and honeycomb structures followed by the nozzle-type flow inlet section to the pipe. This entire inlet flow arrangement also influenced the local wall shear stress at the location where the flows were triggered. However, the flow properties at the location of triggering were not well known and it was therefore decided to carry out an additional set of experiments for which the flow is fully developed at the location of triggering. Triggering was carried out with accurately manufactured ring-type obstacles and the triggering was performed at a location where the flow had reached the state of fully developed laminar pipe flow. Hence, in this set of experiments, the flow conditions at the triggering location were the same. To achieve this, the test rig in figure 1 was employed but the triggering location was set at pipe lengths of $L=5$ and $8 \mathrm{~m}$, at which the flows had reached fully developed states up to $R e \approx 5900$ and 9400 , respectively, as equation (3.1) indicates. Thereafter, a pipe length of $L=2 \mathrm{~m}$ was added to ensure that the triggered flows developed into slugs and puffs as described in $\S \S 4$ and 5. The hot-wire measurements were carried out at the end of this pipe to detect the first slug or puff formation as an indication that the critical Reynolds number of transition had been reached. 


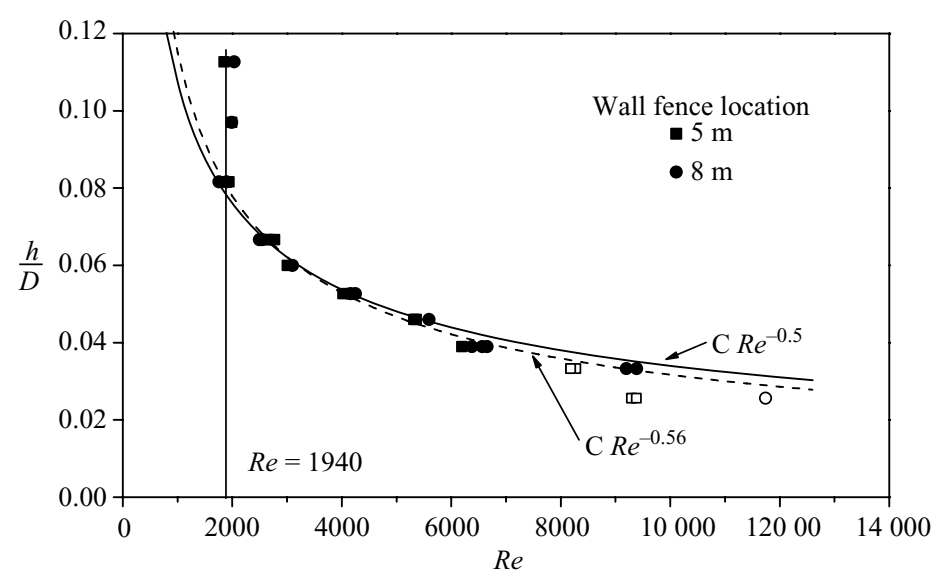

FIGURE 22. Dependence of normalized ring fence height, $h / D$, where $D$ is the pipe diameter, on the critical Reynolds number for fully developed pipe flow at triggering locations $L=5$ and $8 \mathrm{~m}$.

For triggering the flow, nine different ring-type obstacles were laser manufactured with high precision and were placed at the triggering positions described above. Thereafter, and for each obstacle, the mass flow rate was gradually increased until the flow started to show the first disturbances indicating its transition to turbulence. The relationship between the detected critical Reynolds numbers and normalized ring fence height $(h / D)$ for pipe lengths of $L=5$ and $8 \mathrm{~m}$ are shown in figure 22, the C indicating constant values in the figure. The results reflect the following:

(i) With the way in which the experiments were performed, the detected critical Reynolds number for each ring obstacle was that for which the triggered disturbances had not decayed by the end of the pipe.

(ii) According to Jovanović \& Pashtrapanska (2004), who looked at the laminarto-turbulent transition, the following relationship holds:

$$
\left(R e_{\lambda}\right)_{c r i t}=10 \sqrt{5} \sim\left(R e_{D}\right)_{c r i t}^{1 / 2} \sim \frac{D}{\lambda} \sim \frac{D}{h},
$$

where $\lambda$ and $R e_{\lambda}$ are the Taylor microscale and the Reynolds number based on the intensity and length scale of the disturbances, respectively. This readily suggests that

$$
\frac{h}{D} \sim \frac{1}{(\operatorname{Re})_{c r i t}^{1 / 2}} .
$$

(iii) The experimental findings and the $(R e)_{\text {crit }}^{-1 / 2}$ relationships are plotted in figure 22 . The small discrepancy between the predicted $h / D-(R e)_{\text {crit }}$ relationships and the experimental findings is due to the remaining experimental scatter of one data set.

The minimum critical Reynolds number which can be triggered with ring-type obstacles is $(R e)_{\min }=1940$, as the vertical line in figure 22 shows. This lower value critical Reynolds number is in very close agreement with the theoretically obtained $(R e)_{\min }$ deduced by Jovanović \& Pashtrapanska (2004). They investigated theoretically the critical Reynolds number by considering the balance between production and dissipation terms in the turbulence dissipation equation and for pipe flow they obtained in this way $(R e)_{c r i t}=1930$. 


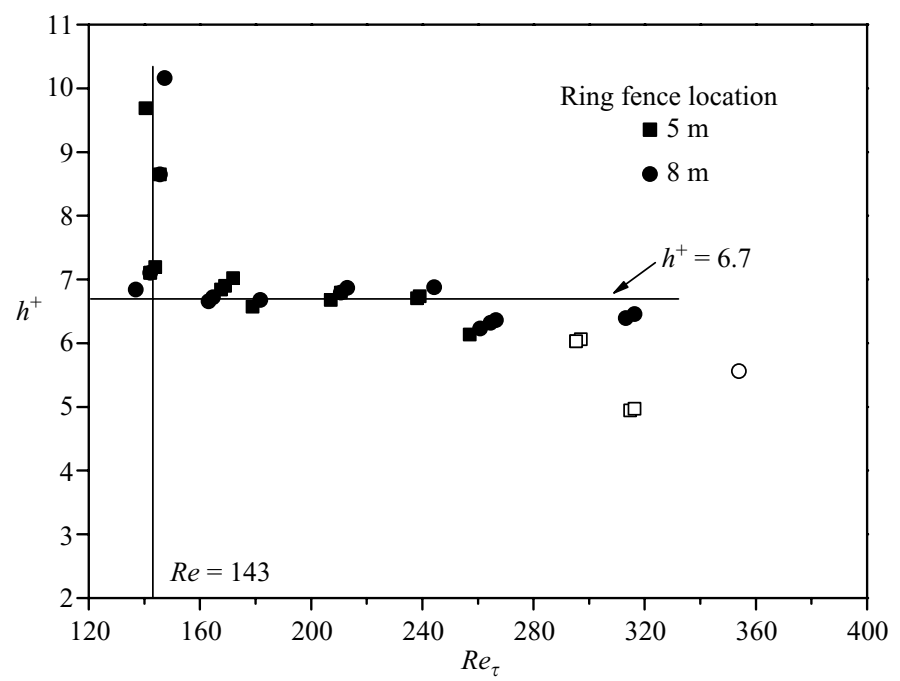

FIGURE 23. Dependence of non-dimensionalized ring fence height on a non-dimensionalized Reynolds number for fully developed pipe flow at triggering locations $L=5$ and $8 \mathrm{~m}$. Open squares and circles are the results of the same experiments but not triggering fully developed flow.

From the results in figure 22, it can readily be deduced that

$$
h^{+}=\frac{h U_{\tau}}{v}=\frac{h}{D} \sqrt{8 R e} \quad \text { since } \quad U_{\tau}=\frac{c_{f}}{2} \tilde{U} .
$$

Taking into account that $h / D \sim(R e)^{-1 / 2}$ suggests that $h^{+}=$constant, i.e. is independent of $R e_{\tau}$. This is shown in figure 23 to be in good agreement with our experimental findings. Other values for the exponent in the Reynolds number dependent relationship for different disturbance amplitudes are given in the literature, e.g. Trefethen et al. (2000), Hof et al. (2003) and Peixinho \& Mullin (2007).

\section{Conclusion, final remarks and outlook}

A special test facility was developed to investigate experimentally the laminarto-turbulent transition of pipe flows. This facility can deterministically create disturbances which develop into puffs and slugs. This is described in the paper, as is the way in which the experiments and measurements should be carried out to study the properties of slugs and puffs. In particular, the results show how slugs and puffs develop along the pipe. The information was obtained by measuring the time variation of the longitudinal velocity at the axis of the pipe outlet cross-section using different lengths of pipes. In a first set of experiments, the development of slugs was studied and the results showed how the slugs expand along the pipe, as a result of differences in the propagation velocities of the front and back edges of the slugs. Second, the development of puffs was investigated with different pipe lengths taking the Reynolds number into account. At fixed Reynolds number, at some distance from the entrance, the structure of puffs and slugs seems to be independent of the nature of the disturbances which created them. At lower Reynolds numbers, puffs form and remain nearly unchanged along a pipe. However, when the Reynolds number of the flow is increased, the puffs are characterized by one or more splits and for sufficiently high Reynolds numbers, the puffs merge into slugs and start to expand as slugs do in 
pipe flows. The centreline velocity at the front edge of puffs changes gradually which also occurs for splitting puffs but not slugs. The present paper provides a very detailed morphology of equilibrium puffs and the mechanical view point of puff splitting and slugs as a function of the Reynolds number.

A last set of experiments was carried out in which the triggering location was moved downstream of the pipe inlet, where the flow had reached the fully developed state of laminar pipe flow. The findings in these investigations suggest the following:

(i) The ring-type obstacle height, normalized with the pipe diameter, required to trigger the laminar-to-turbulent transition of a pipe flow changes with Reynolds number as $\sim(R e)_{\text {crit }}^{-1 / 2}$.

(ii) On plotting the experimental findings as $h^{+}\left(R e_{\tau}\right)$, it is observed that $h^{+}=$ constant. The amplitude perturbation, $h^{+}$, is independent of $R e_{\tau}$. This can be deduced by similarity considerations.

(iii) The investigation shows that the minimum critical Reynolds number required to cause transition is $(R e)_{\min }=1940$, below which obstacle-triggered puffs and slugs cannot be maintained over long pipe lengths. This minimum critical Reynolds number $(R e)_{\min }=1940$ turns out to be very close to the value theoretically deduced by Jovanović \& Pashtrapanska (2004) as $(R e)_{\text {crit }}=1930$.

It is suggested that more experimental studies are needed to deepen the understanding of the flow behaviour in the regime of the laminar-to-turbulent transition of pipe flows. The theory of travelling waves and the possibility that chaos occurs around them (see e.g. Kerswell 2005 and Eckhardt et al. 2007) is an important development in understanding transition. However, more numerical investigations are needed for a complete understanding of the laminar-to-turbulent transition of pipe flows. Also, transitional flows warrant more investigation in order to show the dependence of transition on the pipe diameter and compliance with many results that are available in the literature.

Special thanks are due to Dr Jovan Jovanovic for the valuable discussions. The authors are grateful to the LSTM workshop, especially Mr Jürgen Heubeck, for support during construction of the test facilities. The authors further acknowledge especially Professor Dr Antonio Delgado for his generous support so that the present investigations could be conducted at LSTM-Erlangen. The research stay of G. B. was supported by a DAAD-DST, PPP program.

\section{REFERENCES}

BANDyopadhyay, P. R. 1986 Aspects of equilibrium puff in transitional pipe flow. J. Fluid Mech. 163, 439-458.

Darbyshire, A. G. \& Mullin, T. 1995 Transition to turbulence in constant-mass-flux pipe flow. J. Fluid Mech. 289, 83-114.

Draad, A. A., Kuiken, G. \& Nieuwstadt, F. T. M. 1998 Laminar-turbulent transition in pipe flow for newtonian and non-newtonian fluids. J. Fluid Mech. 377, 267-312.

Durst, F., Heim, U., ÜnSAL, B. \& Kullik, G. 2003 Mass flow rate control system for time-dependent laminar and turbulent flow investigations. Meas. Sci. Technol. 14, 893-902.

Durst, F., Ray, S., Unsal, B. \& Bayoumi, O. A. 2005 The development lengths of laminar pipe and channel flows. J. Fluids Engng 127, 1154-1160.

Durst, F. \& Ünsal, B. 2006 Forced laminar to turbulent transition of pipe flows. J. Fluid Mech. 560, 449-464.

Eckhardt, B., Schneider, T. M., Hof, B. \& Westerweel, J. 2007 Turbulent transition pipe flow. Annu. Rev. Fluid Mech. 39, 447-468. 
FAIsst, H. \& ECKhardt, B. 2004 Sensitive dependence on initial conditions in transition to turbulence in pipe flow. J. Fluid Mech. 504, 343-352.

Hof, B., Juel, A. \& Mullin, T. 2003 Scaling of the turbulence transition threshold in a pipe. Phys. Rev. Lett. 91, 244502, 1-4.

Jovanović, J. \& PASHTRAPANSKa, M. 2004 On the criterion for the determination transition onset and breakdown to turbulence in wall-bounded flows. J. Fluids Engng 126, 626-633.

KeRSWELL, R. R. 2005 Recent progress in understanding the transition to turbulent in a pipe. Nonlinearity 18, R17-R44.

Lindgren, E. R. 1969 Propagation velocity of turbulent slugs and streaks in transition pipe flow. Phys. Fluids 12, 418-425.

Mullin, T. \& Peixinho, J. 2006 Transition to turbulence in pipe flow. J. Low. Temp. Phys. 145, 75-89.

Peixinho, J. \& Mullin, T. 2006 Decay of turbulence in pipe flow. Phys. Rev. Lett. 96, 094501. $1-4$.

Peixinho, J. \& Mullin, T. 2007 Finite-amplitude threshoulds for transition in pipe flow. J. Fluid Mech. 582, 169-178.

REYNOLDS, O. 1883 An experimental investigation of the circumstances which determine whether the motion of water shall be direct of sinuous, and the law of resistance in parallel channels. Philos. Trans. R. Soc. Lond. A 174, 935-982.

RotTA, J. 1956 Experimenteller beitrag zur entstehung turbulenter strömung im rohr. Ing-Arch. 24, $258-281$.

Rubin, Y., Wygnanski, I. J. \& HaRitonidis, J. H. 1980 Further observations on transition in pipe. In Proc. IUTAM Symp. Stuttgart, FRG, pp. 19-26. Springer.

Trefethen, L., Chapman, S., Henningson, D., Meseguer, A., Mullin, T. \& Nieuwstadt, F. 2000 Threshold amplitudes for transition to turbulence in a pipe. http://arXiv.org/abs/physics/ 0007092.

Van Doorne, C. W. H., Hof, B., Nieuwstadt, F. T. M., Westerweel, J. \& Wieneke, B. 2007 Investigation of turbulent puffs in pipe flow with time-resolved stereoscopic PIV. Exps. Fluids 42, 259-279.

Willis, A. P. \& Kerswell, R. R. 2007 Critical behavior in the relaminarization of localized turbulence in pipe flow. Phys. Rev. Lett. 98, 014501, 1-4.

Wygnanski, I. J. \& Champagne, F. H. 1973 On transition in a pipe. Part 1. The origin of puffs and slugs and the flow in a turbulent slug. J. Fluid Mech. 59, 281-351.

Wygnanski, I. J., Sokolov, M. \& Friedman, D. 1975 On transition in a pipe. Part 2. The equilibrium puff. J. Fluid Mech. 69, 283-304. 